\title{
NFATc3 deficiency reduces the classical activation of adipose tissue macrophages
}

\author{
Li Hu1, Fengli He1, Meifeng Huang1, Meihua Peng1, Zhiguang Zhou1, Feng Liu1,2 and Yan-Shan Dai1, \\ 'Department of Metabolism and Endocrinology, Metabolic Syndrome Research Center, Key Laboratory of Diabetes Immunology, Ministry of Education, \\ National Clinical Research Center for Metabolic Disease, The Second Xiangya Hospital, Central South University, Changsha, Hunan, China \\ 2Department of Pharmacology, University of Texas Health Science Center at San Antonio, San Antonio, Texas, USA \\ Correspondence should be addressed to Y-S Dai: ydai88@csu.edu.cn \\ ${ }^{\dagger}($ Y-S Dai is now at Bristol-Myers Squibb Company, Princeton, New Jersey, USA)
}

\begin{abstract}
Nuclear factors of activated T cells (NFAT) c3 have a prominent role in the regulation of proinflammatory factors in immune cells. The classically activated M1 macrophages are key players in the initiation and maintenance of adipose tissue (AT) inflammation. The role of NFATC3 in obesity and AT inflammation is unknown. We set out to determine how deficiency of NFATC3 effected macrophage polarization, inflammation and insulin resistance in visceral AT of high-fat diet (HFD)-fed mice. Nfatc3-/- and WT mice were fed a HFD for 8-17 weeks. Epididymal white AT (eWAT) F4/80(+) cells were characterized by fluorescence-activated cell sorting and quantitative RT-PCR. Results showed that Nfatc3-/- mice developed HFD-induced obesity similar to WT mice, but insulin sensitivity and glucose tolerance were improved, and liver fat accumulation was reduced in Nfatc3-/- mice compared to WT control mice. Moreover, M1 macrophage content and proinflammatory factors were reduced, whereas the alternatively activated M2 macrophage content was increased in eWAT of HFD-fed Nfatc3-/- mice compared to that of WT mice. In addition, eWAT insulin signaling was improved in HFD-fed Nfatc3-/- mice. Importantly, after bone-marrow-derived macrophages had been isolated from Nfatc3-I- mice and cultured in vitro, treatment of these cells with interferon- $\gamma$ and lipopolysaccharide resulted in reduction of M1 inflammatory markers, suggesting that NFATC3 promoted M1 polarization by a cell-autonomous mechanism. The results demonstrated that NFATC3 played an important role in M1 macrophage polarization, AT inflammation and insulin resistance in response to obesity through transcriptional activation of proinflammatory genes.
\end{abstract}

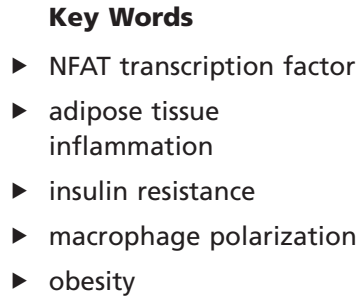

Journal of Molecular Endocrinology (2018) 61, 79-89

\section{Introduction}

Obesity and type 2 diabetes mellitus are associated with low-grade, chronic inflammation and macrophage infiltration in adipose tissue (AT) (Weisberg et al. 2003, Hotamisligil 2006, 2017, Lumeng \& Saltiel 2011, Kusminski et al. 2016, Deng et al. 2017). A number of studies have demonstrated that inhibiting macrophage infiltration in white AT can improve glucose tolerance in the context of obese mice (Osborn \& Olefsky 2012, Aouadi et al. 2013). Also, reduction of macrophage infiltration and inflammation-related gene expression in white AT are associated with weight loss in obese humans (Clement et al. 2004, Cancello et al. 2005). 
Classically activated M1 macrophages are activated by $\mathrm{T}$ helper (Th)- 1 cytokines, such as interferon- $\gamma$ (IFN $\gamma$ ) and lipopolysaccharide (LPS). By contrast, the alternatively activated M2 macrophages are activated with interleukin-4 (IL-4) and interleukin-13 (IL-13). The proinflammatory cytokines IFN $\gamma$, tumor necrosis factor alpha (TNF $\alpha)$ and interleukin-1 beta (IL-1 $\beta$ ) are implicated in disrupting insulin signaling (Mantovani et al. 2004, De Taeye et al. 2007, Harford et al. 2011). The activation of classically activated M1 macrophages and the reduction of antiinflammatory M2 macrophages have been implicated in the development of AT inflammation and insulin resistance (Gordon 2003, Lumeng et al. 2007a, Mosser \& Edwards 2008, McLaughlin et al. 2017). However, the molecular mechanisms underlying how macrophage infiltration was regulated in obesity-induced inflammation in white AT remain to be completely elucidated.

The transcription factors STAT1, STAT6, C/EBPb, IRF-4, IRF5, PPAR $\gamma$ and GATA6 regulate transcriptional programs that control M1/M2 macrophage polarizations (Lawrence \& Natoli 2011, Okabe \& Medzhitov 2014). Candidate regulators can be identified by analysis of promoters of M1-specific genes in macrophages, and among these, IFN $\gamma$ (Sica et al. 1997), TNF (Falvo et al. 2000), IL12p40 (Zhu et al. 2003) and iNOS (Ranjan et al. 2014) promoters are regulated by nuclear factor of activated T cells (NFAT). NFAT family consists of five members, NFATc1, NFATc2, NFATc3 (aka NFAT4), NFATc4 (aka NFAT3) and NFAT5, and they have been implicated in regulating transcription of a number of inducible genes in immune cells (Rao et al. 1997, Crabtree \& Olson 2002, Hogan et al. 2003). The production of several $\mathrm{T}$ cell-derived cytokines including IL-2, IFN $\gamma$ and IL-4 were significantly diminished in T cells lacking both NFATc1 and NFATc2 (Peng et al. 2001). CD4+ Th1 cells have been implicated as positive regulators of AT inflammation via production of IFN $\gamma$, which contributes to the activation of M1 macrophages (Deng et al. 2017). Depletion of Th1 cells with CD3-specific antibodies protected mice from HFD-induced AT inflammation and insulin resistance (Winer et al. 2009). However, mice lacking NFATc3 produce normal levels of serum IL-4 and IFN $\gamma$ (Oukka et al. 1998), but whether their levels change in obese ATs is unknown. In transgenic mice expressing constitutively active NFATc 3 in T cells, NFATc3 enhances expression of Th1 cytokine IFN $\gamma$ and TNF $\alpha$ but suppresses that of Th2 cytokine IL-4 (Chen et al. 2003). NFATc3 and NFATc4 are required for the Toll-like receptor (TLR)activated innate inflammatory response and cytokine induction in macrophages (Minematsu et al. 2011). In macrophages responding to LPS stimulation, NFATc3 undergoes rapid nuclear translocation, whereas NFATc2 and NFATc 4 are localized to the nucleus and NFATc1 is localized to the cytosol, constitutively, suggesting that NFATc3 is the only member rapidly activated by LPS stimulation (Ranjan et al. 2014). LPS-induced NFATc3 in turn directly activates iNOS expression in bone marrowderived macrophages (BMDMs) (Ranjan et al. 2014).

However, it remains unclear whether NFATc3 participates in immune dysfunction causing chronic inflammation and insulin resistance in diet-induced obesity. We showed that reduced M1 macrophage polarization in AT and improved insulin sensitivity in Nfatc3-/- mice fed with HFD. We had identified NFATc3 as a positive regulator of obesity-induced inflammation and insulin resistance. This work was presented at Keystone Symposia in Vancouver, Canada in 2014.

\section{Materials and methods}

Nfatc3-/- mice that were backcrossed to C57BL/6 mice as described before (Oukka et al. 1998) were obtained from Jackson Laboratory (Stock number 010589). Nfatc3+/heterozygous mice were bred to generate age-matched Nfatc3-/- and WT littermates. All mice were maintained in the Specific Pathogen Free (SPF) mice facility of Second Xiangya Hospital under a 12-h light/darkness cycle. At age 6 weeks, male mice were fed either a normal diet (ND, SLA-COM) containing $4 \%$ (wt/wt) total lipids or a HFD (Research Diets) containing $60 \% \mathrm{kcal}$ fat for different weeks. Total body fat content was measured by nuclear magnetic resonance with the Minispec Mq7.5 (Bruker, Karlsruhe, Germany). All animal experiments were performed according to the protocols approved by the Animal Care Research Committee of Second Xiangya Hospital of Central South University. Anti-phosphoAkt (S473) and anti-beta-actin antibody were from Cell Signaling Technology.

\section{Intraperitoneal glucose tolerance tests (IGTTs) and insulin tolerance tests (ITTs)}

IGTTs were carried out with mice that were subjected to overnight (10-12h) food withdrawal. Blood samples were taken from the tail vein before $(0 \mathrm{~min})$ and 15,30 , 60,90 and $120 \mathrm{~min}$ after glucose administration ( $2 \mathrm{~g}$ of glucose/kg body weight). For insulin tolerance (sensitivity) tests, human insulin (0.75 U/kg; Eli Lilly) was administered intraperitoneally to mice that had fasted for $4 \mathrm{~h}$. Blood samples were taken from the tail vein before $(0 \mathrm{~min})$ and 15, 30, 60 and $90 \mathrm{~min}$ after insulin administration. 
Blood glucose levels of samples were determined using an automated blood glucose meter (Roche).

\section{RNA extraction and gene expression}

RNA from cells or mouse tissues were homogenized in TRIzol (Invitrogen) and then followed by purification and DNase I digestion to remove genomic DNA contamination with RNAeasy column (Qiagen) according to the manufacturer's protocol. After reverse transcription, gene expression was quantified with ABI 7900HT Fast RealTime PCR System (Applied Biosystems). Relative gene expression levels were calculated by the use of the $\Delta \Delta \mathrm{Ct}$ method with $18 \mathrm{~S}$ or GAPDH being used as the reference gene (Luo et al. 2014). PCR primer sequences will be available upon request.

\section{Western blots}

Cells or tissues were lysed in lysis buffer $(150 \mathrm{mM} \mathrm{NaCl}$, $0.5 \%$ NP-40, $0.1 \%$ SDS and $50 \mathrm{mM}$ Tris-Cl, $\mathrm{pH} 7.5$ ) with $1 \mathrm{mM}$ PMSF and protease inhibitor cocktail (Sigma). The cell lysates were separated by 10\% SDS-PAGE gels, transferred to a nitrocellulose membrane (Millipore). The membranes were incubated with the primary antibodies anti-AKT, anti-phospho-Akt (S473) and anti-beta-actin, respectively, followed by horseradish peroxidase-labeled donkey anti-rabbit or donkey anti-mouse antibodies. Immunoreactive proteins were visualized with the Molecular Imager Chemi Doc XRS Western Blot Detection System (BIO-RAD), and bands were quantified with Image Lab software as described before (Meng et al. 2017).

\section{Hematoxylin and eosin staining and Oil Red O staining}

H\&E staining and Oil Red $\mathrm{O}$ staining were performed as previously described (Liu et al. 2012). Briefly, for $\mathrm{H} \& \mathrm{E}$ staining, liver tissues from HFD-fed Nfatc3-/- and WT mice were fixed in $10 \%$ formalin solution for $24 \mathrm{~h}$ and then embedded in paraffin. Liver tissue sections $(7 \mu \mathrm{m}$ thick) were stained with hematoxylin-eosin. For Oil Red O staining, liver tissues from HFD-fed Nfatc3-/- and WT mice were flash-frozen by liquid nitrogen. Liver tissue sections ( $5 \mu \mathrm{m}$ thick) were stained with Oil Red O.

\section{Measurement of IFN $\gamma$ protein levels}

Nfatc3-/- and WT control mice, both on HFD, were killed and epididymal fat pad was isolated immediately and were cultured at $37^{\circ} \mathrm{C}$ in a humidified incubator $\left(5 \% \mathrm{CO}_{2}\right)$ overnight. The supernatant IFN $\gamma$ levels were measured with a mouse IFN $\gamma$ ELISA kit from Dakewe Biotech Co.

\section{Isolation of adipose stromal vascular cells}

Mouse primary stromal vascular fractions (SVFs) were isolated and cultured as previously described (Liu et al. 2014). Briefly, subcutaneous white fat depots from Nfatc3-/- or WT control mice (both on HFD) were isolated and minced and then digested by type II collagenase (Sigma-Aldrich). The digested tissue was filtered through a $100 \mathrm{~mm}$ nylon screen. The collected cells were centrifuged for 5 min and suspended in PBS and used for flow cytometry analysis.

For AKT phosphorylation study in AT and AT explants were incubated at $37^{\circ} \mathrm{C}$ in a humidified incubator $(5 \%$ $\mathrm{CO}_{2}$ ) with or without $100 \mathrm{nM}$ insulin in RPMI 1640 medium without serum for $20 \mathrm{~min}$. The AT lysates were subjected to Western blot analysis with anti-phospho-AKT (473) antibody.

\section{Flow cytometry analysis}

SVF cells obtained from eWAT of HFD-fed mice were blocked with rat anti-mouse CD16/CD32 in flow cytometry buffer (PBS; 2\% FBS, $25 \mathrm{mM}$ HEPES, $5 \mathrm{mM}$ EDTA) for $15 \mathrm{~min}$ on ice. For M1 or M2 macrophages, cells were stained with anti-CD11c or anti-CD206 and anti-F4/80 (antibody for flow cytometry were all from Biolegend, San Diego, CA, USA) in flow cytometry buffer for $30 \mathrm{~min}$ on ice protected from light. For CD4+ helper T cell subsets, CD4 and IFN $\gamma$ antibodies were used to stain CD4 Th1 cells, and CD4 and IL-4 antibodies were used to stain CD4 Th2 cells. Cells were washed and pelleted at $200 \mathrm{~g}$ for $10 \mathrm{~min}$ before being re-suspended in flow cytometry buffer. Samples were run on a flow cytometer (Biolegend) and analyzed with FlowJo 10 software.

\section{Isolation of BMDMs}

Mouse bone marrow cells were flushed by PBS from the tibia and femur of male mice and differentiated into BMDMs in DMEM media with 20\% FBS and 10\% L929 cell conditioning media as a source of M-CSF as described before (Dai et al. 2008). Cells were cultured for 7 days at $37^{\circ} \mathrm{C} / 5 \% \mathrm{CO}_{2}$, and then were treated with LPS and IFN $\gamma$ or with IL-4, respectively, to differentiate into M1 or M2 macrophages. 


\section{Statistics}

Statistics were performed using the Prism software (GraphPad). Statistical significance of two groups was assessed by unpaired two-tailed Student's $t$-test or oneway ANOVA when more than two groups were compared. A value of $P<0.05$ was considered significance. All data are expressed as the mean \pm S.E.M.

\section{Results}

\section{Deficiency of NFATc 3 improves glucose and insulin tolerance in HFD-fed mice}

To determine the effect of NFATc3 deficiency on glucose metabolism, Nfatc3-/- mice were placed on either a normal diet or HFD. Interestingly, Nfatc3-/- mice gained weight to a similar extent as did their WT counterparts when fed a HFD (Fig. 1B and C). Because Nfatc3-/- mice weighed less than their WT counterparts at the initiation of the study ( $\sim 30 \mathrm{~g}$ vs $\sim 40 \mathrm{~g}$ respectively), Nfatc3-/- mice weighed less than WT mice on a HFD during the study (Fig. 1A). However, in the end of the study, there were no significant differences in the ratio of body fat/body weight between WT and Nfatc3-/- mice (Fig. 1D), indicating that Nfatc3-/- mice had same fat content as WT mice.

To evaluate how NFATc3 deficiency effects glucose homeostasis, we performed GTT and ITT in WT and Nfatc3-/- mice. HFD-induced pronounced glucose intolerance in WT mice, whereas obese Nfatc3-/- mice were partially protected from glucose intolerance (Fig. 1E). Importantly, high-fat feeding decreased insulin-stimulated glucose disposal in WT mice, whereas Nfatc3-/- mice were partially protected from insulin resistance (Fig. 1F). Taken together, these results indicated that deficiency of NFATc3 moderately protected HFD-fed mice from glucose intolerance and insulin resistance.

\section{AT M1 macrophage content and inflammation are decreased in obese Nfatc3-/- mice}

The accumulation of M1 macrophages in AT couples AT expansion with systemic insulin resistance (Weisberg et al. 2003, Lumeng et al. 2007a, McLaughlin et al. 2017). Therefore, we next examined whether deficiency of NFATc3 influences the accumulation of M1 adipose tissue macrophages (ATMs). As shown in Fig. 2A, epididymal fat pad weights were increased in WT and Nfatc3-/mice, both on a HFD, consistent with the observed increase in total body mass. Particularly, the magnitude of epididymal fat pad expansion in obese Nfatc3-/-
A
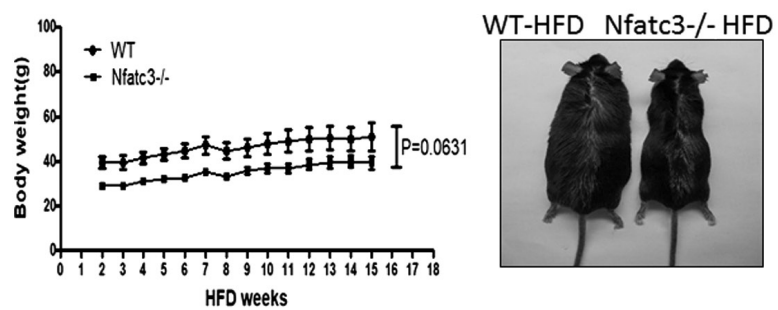

C

D
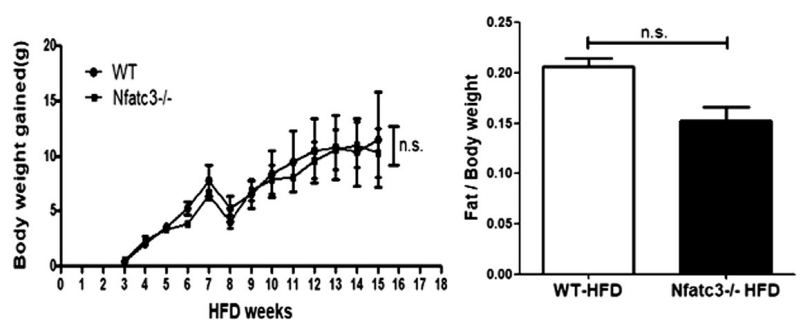

E

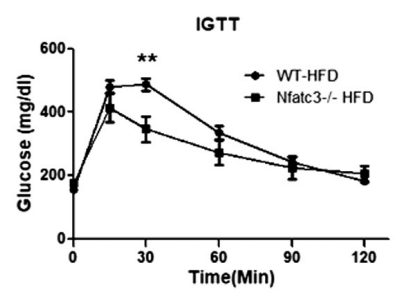

$\mathrm{F}$

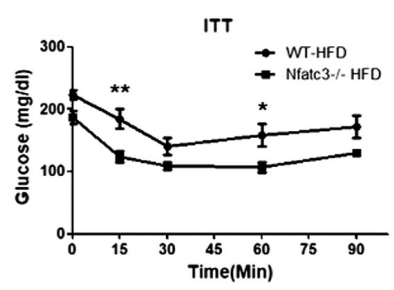

Figure 1

Nfatc3-/- mice were protected from HFD-induced insulin resistance. (A and C) Nfatc3-/- mice and WT counterparts were fed a HFD starting at an age of 6 weeks. Body weights were measured every week; the gained body weights were calculated, which were not significant different between WT and Nfatc3-I- mice. (B) Representative images of mice after a HFD feeding for 16 weeks. (D) At 16 weeks of HFD, body fat accumulation was measured by nuclear magnetic resonance and shown as ratio of total body weight, and there were no significant differences between WT and Nfatc3-I- mice. (E) Glucose tolerance test. Glucose levels were measured after i.p. injection of $2 \mathrm{~g} / \mathrm{kg}$ glucose. ${ }^{*} P<0.01$ vs Nfatc3+/+. $n=6-8$ per group from two independent experiments. (F) Insulin tolerance test. Glucose levels were measured after i.p. injection of $0.75 \mathrm{U} / \mathrm{kg}$ insulin. ${ }^{*} P<0.05$ or $* * P<0.01$ vs $\mathrm{Nfatc} 3+/+. n=6-8$ per group from two independent experiments.

mice was comparable to that of obese WT mice (Fig. 2B). Moreover, the expression of monocyte chemoattractant protein-1 (MCP-1) was significantly decreased in Nfatc3-/- mice relative to WT mice (Fig. 2C). Because M1 ATMs produce inflammatory cytokines that block insulin action (Lumeng et al. 2007b), we next assessed the expression of IL- $1 \beta$ and TNF $\alpha$ in AT and found that their expression levels were significantly decreased in AT of obese Nfatc3-/- mice compared to that of obese WT mice (Fig. 2D and E). Moreover, the secretion of IFN $\gamma$ was decreased in the supernatants of cultured eWAT explants isolated from Nfatc3-/- mice compared to those from WT mice, both on HFD (Fig. 2F). 
A

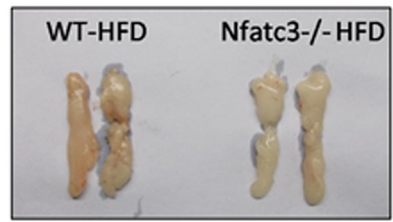

C

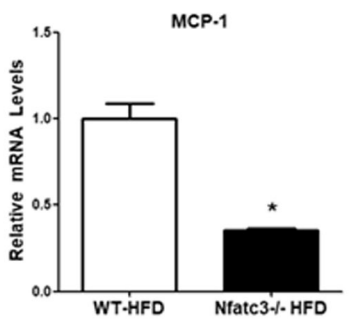

E

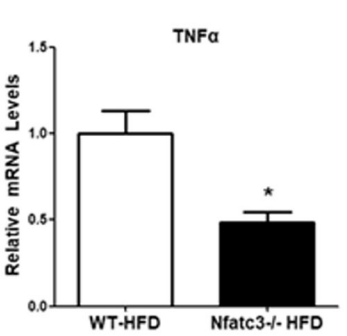

B

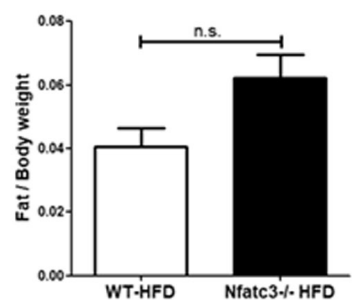

D

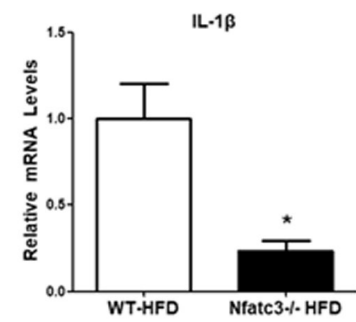

$\mathrm{F}$

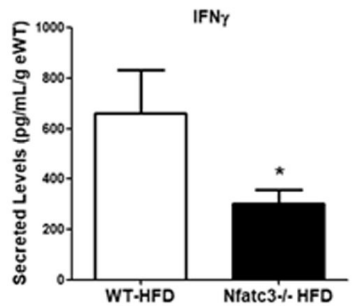

G

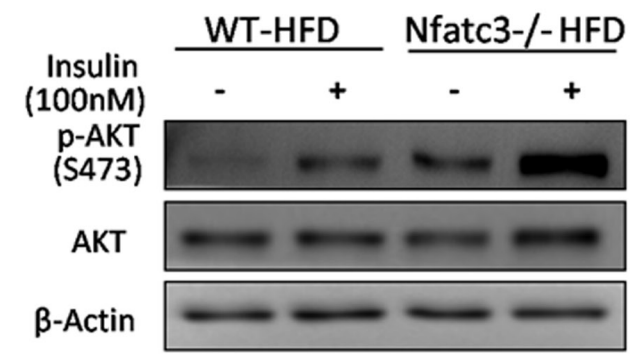

$\mathrm{H}$

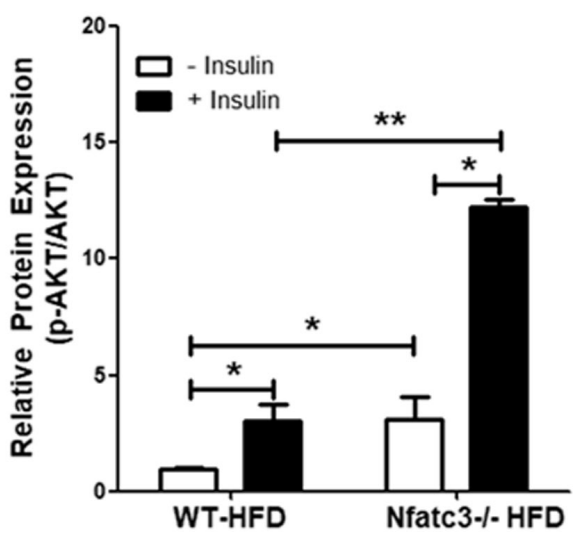

Figure 2

Reduced adipose tissue inflammation and M1 macrophage content in Nfatc3-/- mice on HFD. (A) Representative images of epididymal fat pad (eWAT) from WT and Nfatc3-/- mice on HFD for 16 weeks were shown.

To illuminate how AT insulin signaling was altered by NFATc3 deficiency, we evaluated insulin-stimulated phosphorylation of AKT in eWAT explants of Nfatc3-/mice and WT mice, both on HFD for 17 weeks. Indeed, phosphorylation of AKT was significantly increased in AT of Nfatc3-/- mice compared to that of obese WT mice before and after ex vivo treatment with or without $100 \mathrm{nM}$ insulin for $20 \mathrm{~min}$ (Fig. $2 \mathrm{G}$ and H). These results suggested that HFD-fed Nfatc3-/- mice were protected from insulin resistance in visceral AT presumably due to the reduced levels of IL-1 $1 \beta$, TNF $\alpha$ and IFN $\gamma$.

\section{Deficiency of NFATc3 alters ATM phenotypes}

AT from lean mice contains resident M2 macrophages, whereas the macrophages resident in the expanded AT in obesity are derived either from local proliferation or from circulating monocytes and adopt an M1 phenotype (Lumeng et al. 2008, Shaul et al. 2010, Amano et al. 2014). Because NFATc3 deficiency decreased AT inflammation, we examined whether the phenotype of ATMs was influenced by NFATc3 deficiency. Therefore, we isolated stromal vascular cells from AT of WT or Nfatc3-/- mice (both on HFD) and evaluated the surface expression of CD11c and CD206, markers of M1 and M2 macrophages, respectively, on the total macrophage (F4/80+) population by flow cytometry analysis. HFD feeding prominently increased the population of ATMs expressing CD11c and F4/80 in WT mice but not in Nfatc3-/- mice (Fig. 3C). HFD feeding also increased total F4/80 macrophages and total CD45+ immune cells in WT mice but not in Nfatc3-/- mice (Fig. 3A and C). Conversely, the amount of ATMs expressing F4/80 and CD206 (M2) was increased in Nfatc3-/- mice compared to WT mice (Fig. 3B and D). CD206+ F4/80 macrophages relative to total live cells or to total CD45+ immune cells were both increased in HFD-fed Nfatc3-/- mice (Fig. 3D). Representative dot plots of the F4/80+ ATMs were shown in Fig. 3A and B. We next examined the expression of characteristic M2

(B) eWAT was weighed and the fat-to-body weight ratio was not significantly different between WT and Nfatc3-I- mice. (C, D and E) Gene expressions of MCP-1, IL-1 $\beta$ and TNF $\alpha$ in adipose tissue from HFD-fed Nfatc3-/- and Nfatc3+/+ mice were analyzed by real-time quantitative RT-PCR. ${ }^{*} P<0.05$ vs WT mice. $n=3$ per group. (F) The IFN $\gamma$ concentrations in cultured eWAT supernatants were measured with a mouse IFN $\gamma$ ELISA kit. (G) Insulin-stimulated phosphorylation of AKT in the adipose tissue of Nfatc3-I- and WT mice was determined by Western blot analysis. (H) The relative phosphorylation levels of AKT shown in (G) were quantified. ${ }^{*} P<0.05$ or $* * P<0.01$ vs WT mice. $n=3$ per group. A full color version of this figure is available at https://doi.org/10.1530/ JME-18-0070. 

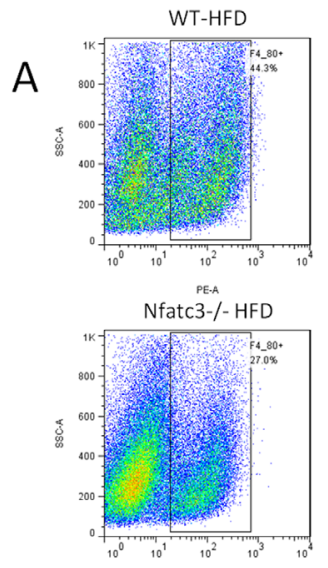

PEAA:F4_BOPE

WT-HFD

B

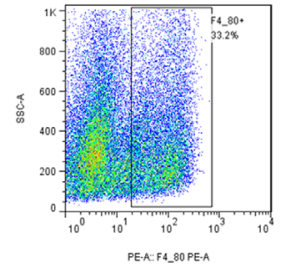

Nfatc3-/- HFD

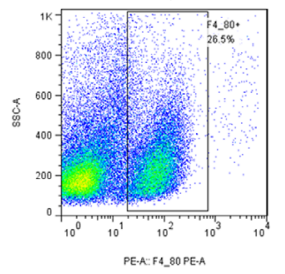

C

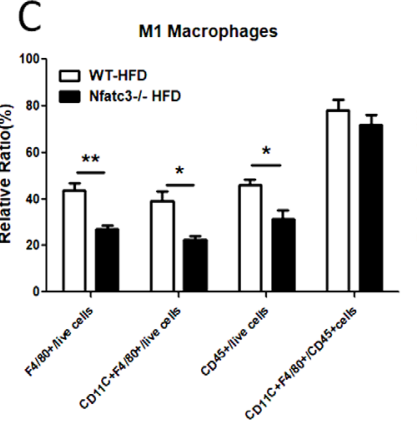

F

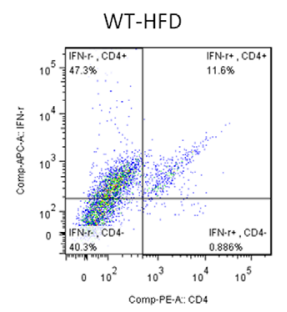

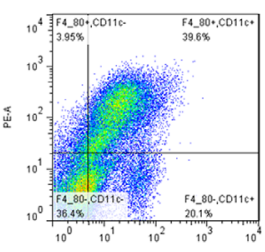

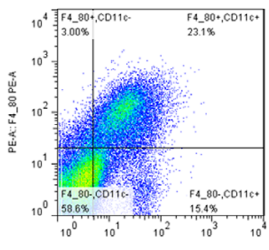

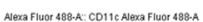

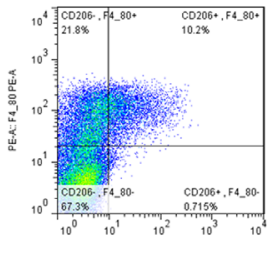

FITCA:CD206 FITCAA

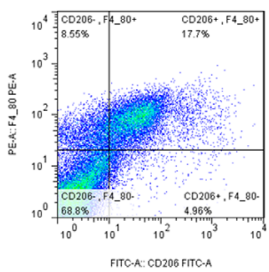

FTTCA: CD206 FTTCA

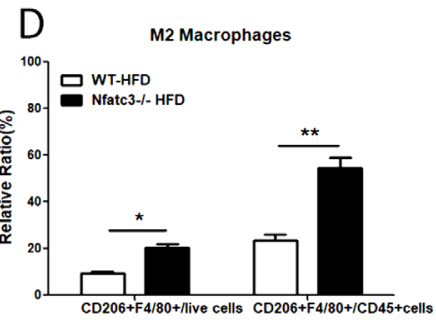

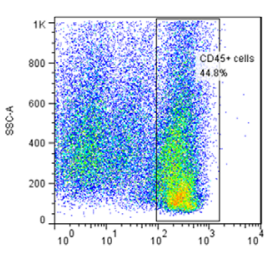

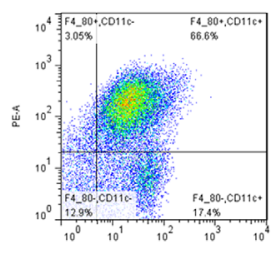

APC.COA-A
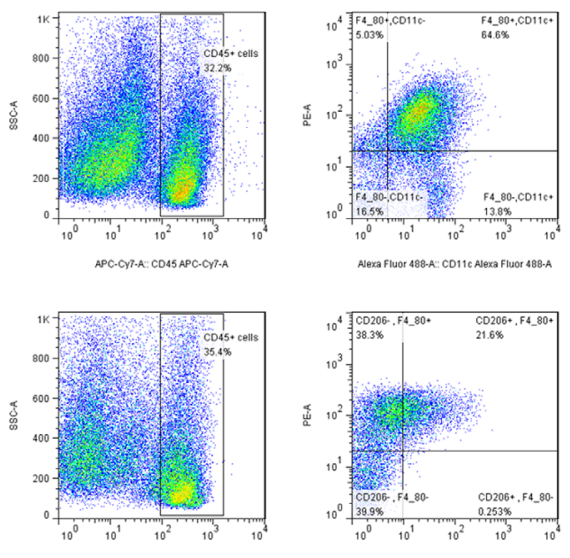

APC.CYTA: CDA5 APCC.CYT.A
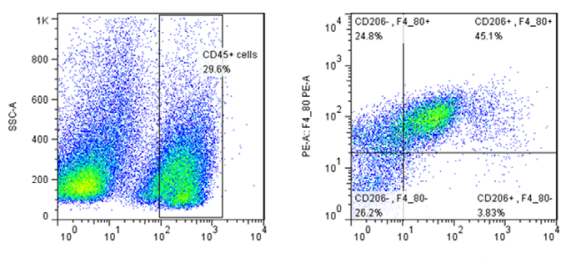

FTTCA: CO206 FTTCA

E

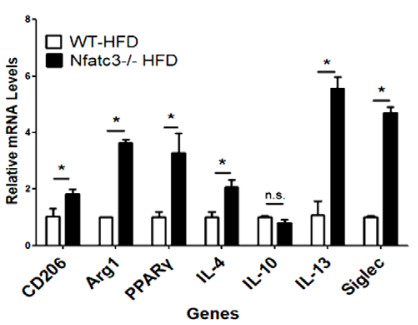

G
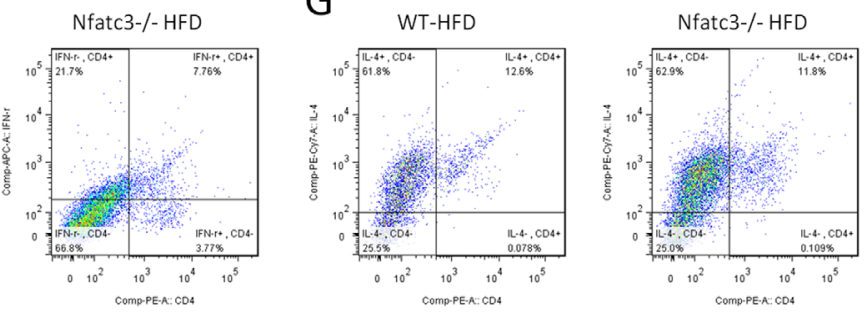

Figure 3

Reduced M1 ATMs and increased M2 ATMs in Nfatc3-I- mice fed a HFD. (A and B) ATMs contents were assessed by flow cytometry in SVF cells obtained from eWAT of HFD-fed Nfatc3-I- and WT mice. Cells were gated for F4/80+ ATMs and analyzed for expression of markers of M1 (CD11c) and M2 (CD206) ATMs. (C and D) Data shown as percentage of CD45+ cells or total live SVF cells. ${ }^{*} P<0.05$ or $* * P<0.01$ vs WT mice. $n=3-4$ per group. (E) Expression analysis of inflammatory genes in adipose tissue from Nfatc3-I- and WT mice both on HFD. SVF cells obtained from eWAT of HFD-fed mice were analyzed by real-time quantitative RT-PCR. ${ }^{*} P<0.05$ vs Nfatc $3+/+. n=3$ per group. (F and G) Cells were gated for CD4+ T cells and analyzed for the expression of IFN $\gamma$ for CD4+ Th1 cells (F) and for the expression of IL-4 for CD4+ Th2 cells (G). 
genes to evaluate how ATM phenotype was influenced by NFATc3 deficiency. The expression of M2 genes Arg1, IL-4 and IL-13 was significantly increased in Nfatc3-/mice compared to WT mice on HFD (Fig. 3E). Together, these results suggested that NFATc3 deficiency prevented polarization of M1 ATMs in obesity and consequently altered the equilibrium between M1 and M2 ATMs.

Since CD4+ Th1 cells have been implicated as positive regulators of AT inflammation via production of IFN $\gamma$ (Winer et al. 2009), we isolated stromal vascular cells from AT of WT or Nfatc3-/- mice (both on HFD) and sorted the surface expression of CD4+ IFN $\gamma$ for CD4 Th1 cells and that of CD4+ IL-4 for Th2 cells on the total CD4+ T cell population by flow cytometry. The results in Fig. 3F showed that CD4+ IFN $\gamma+$ cells accounted for $11.6 \%$ among CD4+ cells in SVF cells of WT mice compared to $7.8 \%$ in those of Nfatc3-/- mice. In contrast, there were no significant differences in CD4+ IL-4+ cells, which accounted for $12.6 \%$ on the total CD4+ T cells of WT SVF compared to $11.8 \%$ on those of Nfatc3-deficient SVF (Fig. 3G). The results suggested that reduction of both CD4+ Th1 cells and IFN $\gamma$ in turn contributed to the reduced M1 macrophages in the AT of HFD-fed Nfatc3-/mice.

\section{Obesity-induced hepatic steatosis and inflammation are improved by NFATc 3 deficiency}

Macrophage-mediated AT inflammation can promote insulin resistance in other insulin-sensitive tissues, such as the liver and skeletal muscle (Olefsky \& Glass 2010, Lackey \& Olefsky 2016). Insulin resistance in the liver can be caused by fatty acid release from AT, resulting in hepatic triglyceride accumulation; also, insulin resistance can be caused by increased inflammatory cytokines produced from the AT (Samuel \& Shulman 2016). Therefore, we tested whether deficiency of NFATc3 improved hepatic steatosis in HFD-fed mice. As shown in Fig. 4A, NFATc3 deficiency markedly alleviated diet-induced steatosis in the liver. Histological analysis of the liver showed an apparent decrease in fat accumulation in Nfatc3-/- mice (Fig. 4B). Accordingly, fat staining with Oil Red O showed a reduction in total hepatic fat content in Nfatc3-/- mice on HFD, compared to obese WT mice (Fig. 4C). Because steatosis can induce hepatic inflammation, we next evaluated whether the inflammatory factors were reduced by NFATc3 deficiency. Indeed, decreased MCP-1 and Tolllike receptor 4 (TLR4) mRNA were observed in the liver in Nfatc3-/- mice compared to WT mice, both on HFD (Fig. 4D and E). It has been shown that TLR4 stimulated
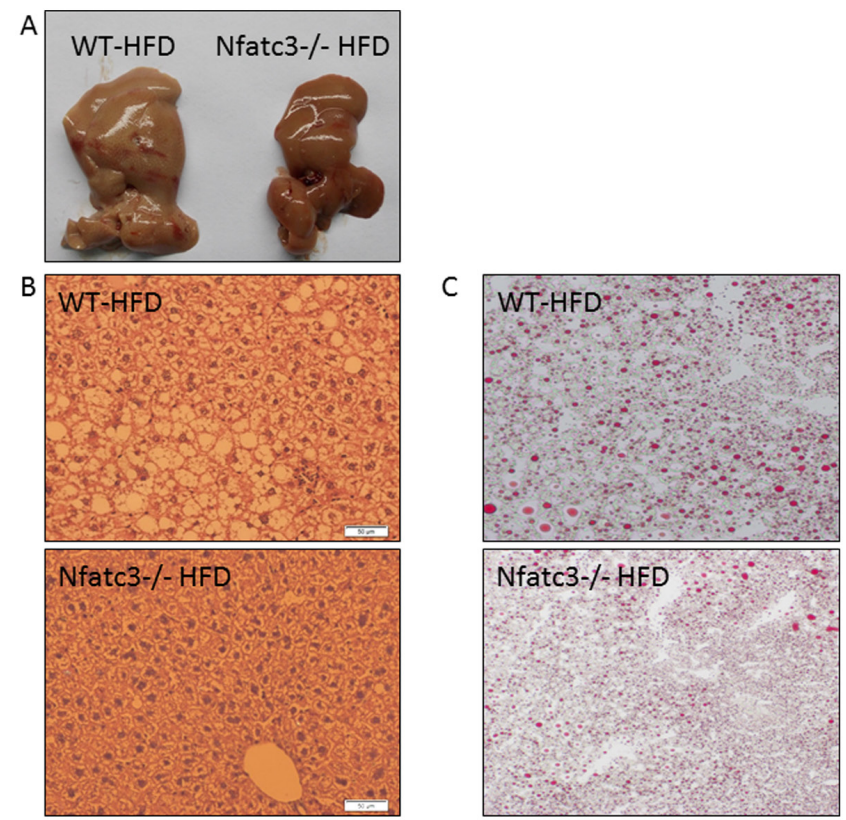

Nfatc $3-1$ - HED

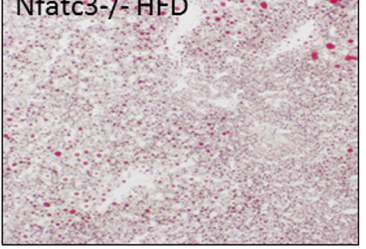

D
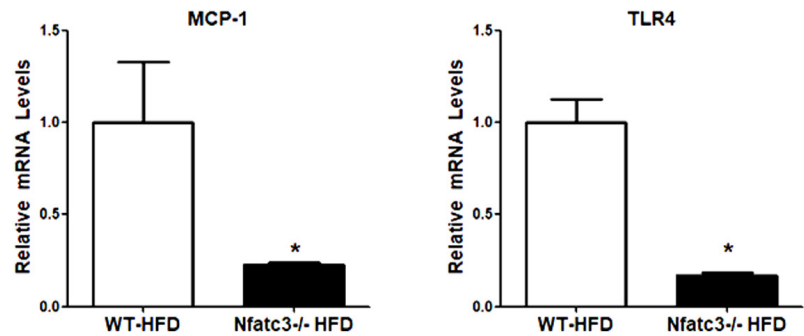

Figure 4

Nfatc3-I- mice were protected from obesity-induced hepatic steatosis and inflammation. (A) Representative images of the livers from WT and Nfatc3-/- mice on HFD for 16 weeks were shown. Hematoxylin and eosin (H\&E)-stained sections (B) and oil red O staining (C) from the liver of HFD-fed Nfatc3-I- and HFD-fed WT mice. Representative images were shown at $20 \times$ magnification. Similar results were seen in four independent samples. (D and E) Gene expressions of MCP-1 and TLR4 in the liver of HFD-fed Nfatc3-/- and HFD-fed WT mice were analyzed by real-time quantitative RT-PCR. ${ }^{*} P<0.05$ vs $N f a t c 3+/+. n=3$ per group.

by LPS drives macrophages to M1 phenotype (Orr et al. 2012).

\section{NFATc3 is required for M1 macrophage activation in BMDMs ex vivo}

The Nfatc3-deleted mouse line that we analyzed was not conditional gene knockout; therefore, in order to understand whether NFATc3 functions in a cellautonomous or non-cell-autonomous manner in AT macrophages, we used an ex vivo system, whereby BMDMs were differentiated into the M1 or M2 lineage. Type 2 cytokines IL- 13 and IL- 4 secreted by adipocytes and eosinophils activate ATMs toward the anti-inflammatory 
M2 phenotype, whereas IFN $\gamma$ activates ATMs to differentiate into the M1 phenotype (McLaughlin et al. 2017). Therefore, BMDMs were isolated from Nfatc3-/and WT mice under normal diet and treated with LPS and IFN $\gamma$ or with IL- 4 to promote M1 or M2 differentiation, respectively. Subsequently, the expression of specific markers of each phenotype was determined by quantitative RT-PCR. Remarkably, BMDMs from Nfatc3-/- mice had a dramatically reduced ability to respond to LPS and IFN $\gamma$, as shown by the lower levels of induced IL-1 $\beta$ and TNF $\alpha$
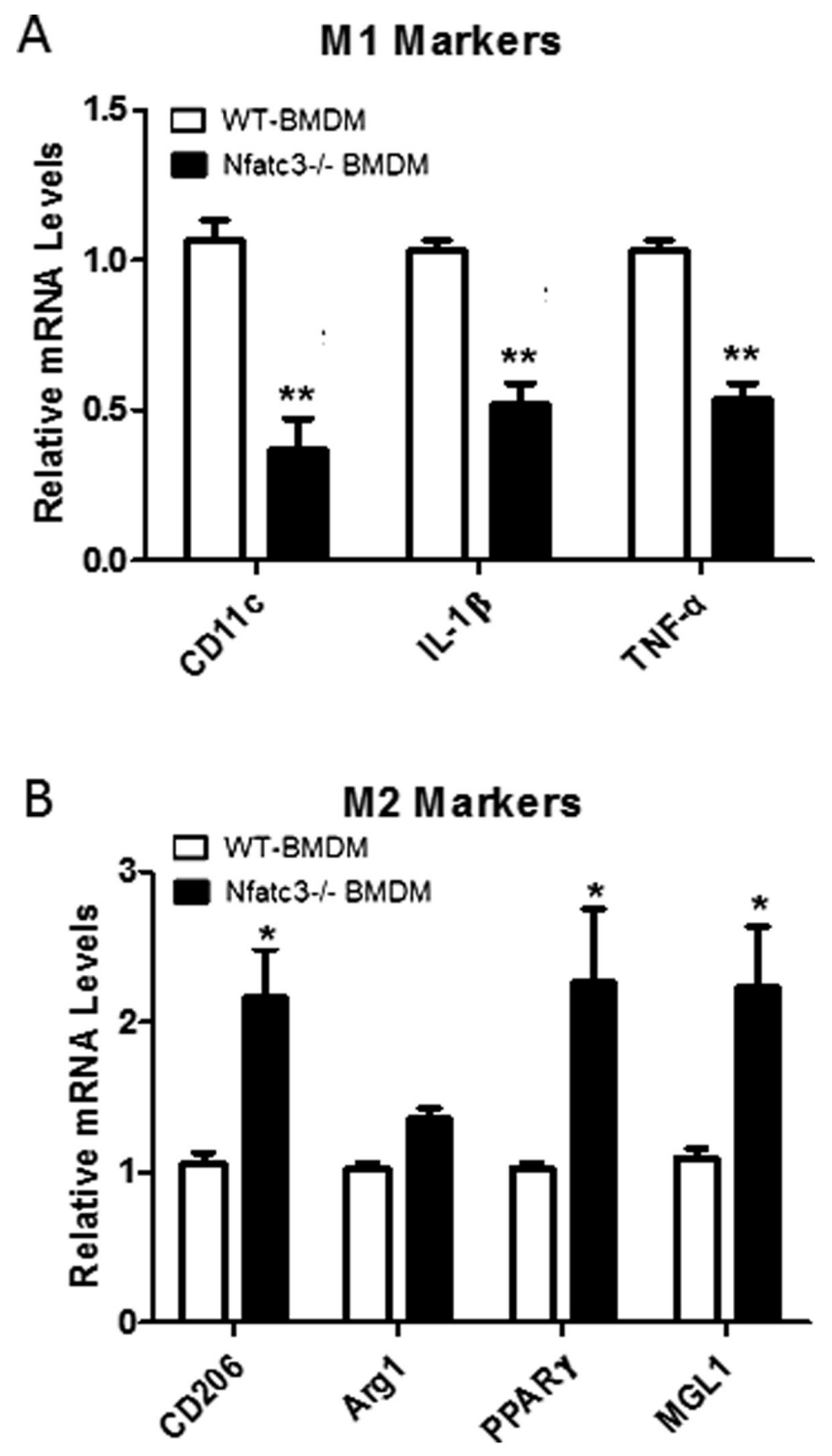

Figure 5

BMDMs from Nfatc3-/- mice were more inclined to differentiate into M2 macrophages. Bone-marrow-derived macrophages (BMDMs) from normal diet-fed Nfatc3-I- and WT mice were differentiated into M1 or M2 lineage. Markers of M1 (A) or M2 (B) macrophages were analyzed by real-time quantitative RT-PCR. ${ }^{*} P<0.05$ or $* * P<0.01$ vs Nfatc $3+/+. n=3$ per group.

(C) 2018 Society for Endocrinology Published by Bioscientifica Ltd. Printed in Great Britain
mRNA, but they were sensitive to IL-4, as demonstrated by higher levels of Arg 1 and PPAR $\gamma$ mRNA (Fig. 5A and B). These results suggested that NFATc 3 was required for the differentiation of macrophages into M1, but not M2, phenotype and therefore was an important transcriptional regulator of proinflammatory factors in macrophage in a cell-autonomous manner.

\section{Discussion}

NFAT family has diverse activities, such as the activation of genes encoding type I interferon (Bao et al. 2016), inflammatory cytokines, including TNF $\alpha$ and IL-2 (Rao et al. 1997, Crabtree \& Olson 2002). Here, we demonstrate a role for NFATc3 in regulating M1 macrophage lineage in ATs in HFD-fed mice. Our results showed reduced CD4+ Th1 cells and IFN $\gamma$ in AT of HFD-fed Nfatc3-/- mice, suggesting a mechanism for macrophage polarization toward M2 phenotype in these mice. Remarkably, when BMDMs isolated from WT or Nfatc3-/- mice were cultured in vitro and treated by LPS and IFN $\gamma$ during their differentiation, the induction of M1-specific cytokines was reduced in BMDMs of Nfatc3-/- mice compared to those of WT control mice, suggesting that NFATc3 regulated M1 macrophage polarization in the macrophage in a cell-autonomous manner. The results documented a previously unrecognized role of NFATc3 in promoting M1 macrophage polarization, inflammation and insulin resistance in obesity.

Obesity promotes the recruitment of monocytes from the bone marrow in part by activating the $\mathrm{C}-\mathrm{C}$ motif chemokine receptor 2 (Ccr2). Deficiency of Ccr2 or its ligand, MCP-1, in mice protects from monocyte infiltration into AT and colonic inflammation (Lumeng et al. 2007a, Odegaard et al. 2007, Kawano et al. 2016). MCP-1 also drives macrophages within the visceral AT to undergo proliferation (Amano et al. 2014). Our results showed reduced MCP-1 in the AT in Nfatc3-/- mice on HFD, suggesting that reduced M1 macrophage polarization in obese ATs in Nfatc3-/- mice may be partly due to reduced MCP-1. NFATc3 is highly expressed on macrophages; previous studies have demonstrated that NFATc3 nuclear localization in macrophages is increased upon LPS stimulation (Ranjan et al. 2014). Moreover, since NFATc3 has been shown to regulate proinflammatory cytokines, including iNOS, IFN $\gamma$ and TNF $\alpha$, our results suggested that NFATc3 promoted M1 macrophage polarization in AT and BMDMs through activation of these cytokines. Although our studies suggested a key role of NFATc3 in mediating tissue recruitment of monocytes in obesity, we had not 
ruled out the possibility that NFATc3 was an important factor in proliferating macrophages at the local tissue level as local proliferation of macrophages also contributes to AT inflammation (Amano et al. 2014). Our studies showed a profound decrease in the activation of inflammatory signaling in AT, liver and BMDMs in Nfatc3-/- mice on HFD; therefore, NFATc3 pathway may play an important role in both recruitment and local proliferation of monocytes and macrophages in these tissues.

Macrophage polarization toward a M1 or M2 state depends generally on cytokines and lipid mediators (Mantovani et al. 2004). A high ratio of inflammatory M1 ATMs (F4/80+CD11c+) to resident M2 ATMs (F4/80+CD206+) is responsible for both adipose tissue as well as systemic insulin resistance (Lumeng et al. 2007a, Shaul et al. 2010). In comparison with obese WT mice, ATMs were considerably decreased in obese Nfatc3-/mice, which was associated with a higher M2/M1 ratio. This observation was confirmed by the identification of higher M2 marker mRNA, including ARG1, in the AT and BMDMs isolated from Nfatc3-/- mice. Because PPAR $\gamma$ regulates alternative activation of macrophages and is associated with increased insulin sensitivity (Odegaard et al. 2007), it is likely that PPAR $\gamma$ caused the higher proportion of M2 macrophages in the AT of Nfatc3-/- mice. Indeed, we found increased PPAR $\gamma$ expression in Nfatc3-deficient BMDMs after IL-4 stimulation. In HFD-fed Nfatc3-/- mice, M1 polarization was largely reduced and insulin signaling was well-maintained in the AT; triglyceride accumulation in the liver was largely reduced. These data suggested that NFATc3 regulated macrophage polarization in the AT and that AT inflammation causes inflammation and insulin resistance in other insulin-sensitive tissues (Rosen \& Spiegelman 2006, Guilherme et al. 2008, Reilly \& Saltiel 2017). Our results also were in line with previous studies that showed lipolysis in AT promotes hepatic triglyceride accumulation and subsequent insulin resistance (Vigouroux et al. 2011, Gan et al. 2015). A duration of high-fat feeding for 17 weeks were chosen because it can induce AT inflammation and insulin resistance in the liver, skeletal muscle and the AT (Kim et al. 2008).

Because IFN $\gamma$ and IL-1 $\beta$ inhibit insulin signaling and promote insulin resistance in adipocytes (McGillicuddy et al. 2009), decreased IFN $\gamma$ and IL-1 $\beta$ in the AT of Nfatc3-/- mice led to the improved insulin sensitivity in Nfatc3-/- mice. In addition, Nfatc3-deficient BMDMs displayed an M2-skewed polarization profile at the mRNA level and exhibited a significantly lower expression of proinflammatory cytokines (TNF $\alpha$ and IL-1 $\beta$ ) in response to LPS and IFN $\gamma$. Therefore, NFATc3 influences macrophage polarization by regulating the expression of polarization-associated genes in macrophages.

Infiltration of $\mathrm{CD} 4+\mathrm{T}$ cells into the $\mathrm{AT}$ and development of insulin resistance transpires 5 weeks after HFD feeding in mice, whereas recruitment of macrophages in AT was delayed until 10 weeks of HFD (Kintscher et al. 2008, Stolarczyk 2017). CD4+ T cells react with antigenic peptides associated with class II major histocompatibility complex (MHC) molecules; adipocytes expressing class II MHC activate CD4+ T cells and increase the leptin-induced secretion of IFN $\gamma$ by CD4+ T cells (Deng et al. 2013). IFN $\gamma$ then activates M1 macrophages, which secrete numerous cytokines and chemokines, including TNF- $\alpha$, IL-1 $\beta$ and MCP-1, causing insulin resistance in adipocytes. M1 macrophages also promote Th1 polarization of CD4+T cells by IL-12 production, reinforcing the positive feedback loop between CD4+Th1 cells and M1 macrophages. In Nfatc3-/- mice on HFD, $\mathrm{CD} 4+\mathrm{IFN} \gamma+$ cells were reduced in the AT compared to WT control, suggesting regulation of recruitment of AT CD4+ Th1 cells by NFATc3. Interestingly, T helper cells can also promote cardiac fibrosis by secretion of cytokines and activation of macrophages (Nevers et al. 2017). In mouse, heart failure model induced by pressure overload, activated Th1 $\mathrm{T}$ cells promote cardiac fibrosis in an IFN $\gamma$-dependent manner by inducing the transition of cardiac fibroblasts into TGF- $\beta$-producing myofibroblasts (Groschel et al. 2017), suggesting a potential mechanism for protection of cardiac hypertrophy and heart failure in Nfatc3-/- mice (Wilkins et al. 2002).

Collectively, these results identify NFATc3 as a regulator of macrophage polarization in AT through activation of macrophage proinflammatory genes in HFDfed mice and shed new insights into the mechanisms by which NFATc3 contributes to macrophage polarization, inflammation and insulin resistance in the AT.

\section{Declaration of interest}

The authors declare that there is no conflict of interest that could be perceived as prejudicing the impartiality of the research reported.

\section{Funding}

This work was supported by grants from the National Natural Science Foundation of China (81270905 to Y D).

\section{Author contribution statement \\ $Y \mathrm{D}$ and $\mathrm{L} \mathrm{H}$ conceived and designed the experiments; $\mathrm{LH}, \mathrm{FH}, \mathrm{M} \mathrm{H}$ and $Y D$ performed the experiments; L H, F H, M H, M P, Z Z, F L and $\mathrm{Y} D$ analyzed the data; $\mathrm{M} \mathrm{P}, \mathrm{ZZ}$ and $\mathrm{FL}$ contributed reagents/materials/ analysis tools; $\mathrm{Y} \mathrm{D}$ and $\mathrm{L} \mathrm{H}$ contributed to the writing of the manuscript.}




\section{Ethical approval}

All applicable international, national and/or institutional guidelines for the care and use of animals were followed. This article does not contain any studies with human participants performed by any of the authors.

\section{References}

Amano SU, Cohen JL, Vangala P, Tencerova M, Nicoloro SM, Yawe JC, Shen Y, Czech MP \& Aouadi M 2014 Local proliferation of macrophages contributes to obesity-associated adipose tissue inflammation. Cell Metabolism 19 162-171. (https://doi. org/10.1016/j.cmet.2013.11.017)

Aouadi M, Tencerova M, Vangala P, Yawe JC, Nicoloro SM, Amano SU, Cohen JL \& Czech MP 2013 Gene silencing in adipose tissue macrophages regulates whole-body metabolism in obese mice. PNAS 110 8278-8283. (https://doi.org/10.1073/pnas.1300492110)

Bao M, Wang Y, Liu Y, Shi P, Lu H, Sha W, Weng L, Hanabuchi S, Qin J, Plumas J, et al. 2016 NFATC3 promotes IRF7 transcriptional activity in plasmacytoid dendritic cells. Journal of Experimental Medicine $\mathbf{2 1 3}$ 2383-2398. (https://doi.org/10.1084/jem.20160438)

Cancello R, Henegar C, Viguerie N, Taleb S, Poitou C, Rouault C, Coupaye M, Pelloux V, Hugol D, Bouillot JL, et al. 2005 Reduction of macrophage infiltration and chemoattractant gene expression changes in white adipose tissue of morbidly obese subjects after surgery-induced weight loss. Diabetes 54 2277-2286. (https://doi. org/10.2337/diabetes.54.8.2277)

Chen J, Amasaki Y, Kamogawa Y, Nagoya M, Arai N, Arai K \& Miyatake S 2003 Role of NFATx (NFAT4/NFATc3) in expression of immunoregulatory genes in murine peripheral CD4+ T cells. Journal of Immunology 170 3109-3117. (https://doi.org/10.4049/ jimmunol.170.6.3109)

Clement K, Viguerie N, Poitou C, Carette C, Pelloux V, Curat CA, Sicard A, Rome S, Benis A, Zucker JD, et al. 2004 Weight loss regulates inflammation-related genes in white adipose tissue of obese subjects. FASEB Journal 18 1657-1669. (https://doi.org/10.1096/fj.04$2204 \mathrm{com})$

Crabtree GR \& Olson EN 2002 NFAT signaling: choreographing the social lives of cells. Cell 109 (Supplement) S67-S79. (https://doi. org/10.1016/S0092-8674(02)00699-2)

Dai Y, Ashraf M, Zuo S, Uemura R, Dai YS, Wang Y, Haider H, Li T \& $\mathrm{Xu}$ M 2008 Mobilized bone marrow progenitor cells serve as donors of cytoprotective genes for cardiac repair. Journal of Molecular and Cellular Cardiology 44 607-617. (https://doi.org/10.1016/j. yimcc.2007.11.011)

De Taeye BM, Novitskaya T, McGuinness OP, Gleaves L, Medda M, Covington JW \& Vaughan DE 2007 Macrophage TNF-alpha contributes to insulin resistance and hepatic steatosis in diet-induced obesity. American Journal of Physiology: Endocrinology and Metabolism 293 E713-E725. (https://doi.org/10.1152/ajpendo.00194.2007)

Deng T, Lyon CJ, Minze LJ, Lin J, Zou J, Liu JZ, Ren Y, Yin Z, Hamilton DJ, Reardon PR, et al. 2013 Class II major histocompatibility complex plays an essential role in obesity-induced adipose inflammation. Cell Metabolism 17 411-422. (https://doi. org/10.1016/j.cmet.2013.02.009)

Deng T, Liu J, Deng Y, Minze L, Xiao X, Wright V, Yu R, Li XC, Blaszczak A, Bergin S, et al. 2017 Adipocyte adaptive immunity mediates diet-induced adipose inflammation and insulin resistance by decreasing adipose Treg cells. Nature Communications $\mathbf{8} 15725$. (https://doi.org/10.1038/ncomms15725)

Falvo JV, Uglialoro AM, Brinkman BM, Merika M, Parekh BS, Tsai EY, King HC, Morielli AD, Peralta EG, Maniatis T, et al. 2000 Stimulusspecific assembly of enhancer complexes on the tumor necrosis factor alpha gene promoter. Molecular and Cellular Biology 20 2239-2247. (https://doi.org/10.1128/MCB.20.6.2239-2247.2000)
Gan L, Xiang W, Xie B \& Yu L 2015 Molecular mechanisms of fatty liver in obesity. Frontiers in Medicine 9 275-287. (https://doi.org/10.1007/ s11684-015-0410-2)

Gordon S 2003 Alternative activation of macrophages. Nature Reviews Immunology 3 23-35. (https://doi.org/10.1038/nri978)

Groschel C, Sasse A, Rohrborn C, Monecke S, Didie M, Elsner L, Kruse V, Bunt G, Lichtman AH, Toischer K, et al. 2017 T helper cells with specificity for an antigen in cardiomyocytes promote pressure overload-induced progression from hypertrophy to heart failure. Scientific Reports 7 15998. (https://doi.org/10.1038/s41598-017-16147-1)

Guilherme A, Virbasius JV, Puri V \& Czech MP 2008 Adipocyte dysfunctions linking obesity to insulin resistance and type 2 diabetes. Nature Reviews Molecular Cell Biology 9 367-377. (https:// doi.org/10.1038/nrm2391)

Harford KA, Reynolds CM, McGillicuddy FC \& Roche HM 2011 Fats, inflammation and insulin resistance: insights to the role of macrophage and T-cell accumulation in adipose tissue. Proceedings of the Nutrition Society 70 408-417. (https://doi.org/10.1017/ S0029665111000565)

Hogan PG, Chen L, Nardone J \& Rao A 2003 Transcriptional regulation by calcium, calcineurin, and NFAT. Genes and Development $\mathbf{1 7}$ 2205-2232. (https://doi.org/10.1101/gad.1102703)

Hotamisligil GS 2006 Inflammation and metabolic disorders. Nature $\mathbf{4 4 4}$ 860-867. (https://doi.org/10.1038/nature05485)

Hotamisligil GS 2017 Inflammation, metaflammation and immunometabolic disorders. Nature 542 177-185. (https://doi. org/10.1038/nature21363)

Kawano Y, Nakae J, Watanabe N, Kikuchi T, Tateya S, Tamori Y, Kaneko M, Abe T, Onodera M \& Itoh H 2016 Colonic proinflammatory macrophages cause insulin resistance in an intestinal Ccl2/Ccr2-dependent manner. Cell Metabolism 24 295-310. (https:// doi.org/10.1016/j.cmet.2016.07.009)

Kim F, Pham M, Maloney E, Rizzo NO, Morton GJ, Wisse BE, Kirk EA, Chait A \& Schwartz MW 2008 Vascular inflammation, insulin resistance, and reduced nitric oxide production precede the onset of peripheral insulin resistance. Arteriosclerosis, Thrombosis, and Vascular Biology 28 1982-1988. (https://doi.org/10.1161/ ATVBAHA.108.169722)

Kintscher U, Hartge M, Hess K, Foryst-Ludwig A, Clemenz M, Wabitsch M, Fischer-Posovszky P, Barth TF, Dragun D, Skurk T, et al. 2008 T-lymphocyte infiltration in visceral adipose tissue: a primary event in adipose tissue inflammation and the development of obesitymediated insulin resistance. Arteriosclerosis, Thrombosis, and Vascular Biology 28 1304-1310. (https://doi.org/10.1161/ATVBAHA.108.165100)

Kusminski CM, Bickel PE \& Scherer PE 2016 Targeting adipose tissue in the treatment of obesity-associated diabetes. Nature Reviews Drug Discovery 15 639-660. (https://doi.org/10.1038/nrd.2016.75)

Lackey DE \& Olefsky JM 2016 Regulation of metabolism by the innate immune system. Nature Reviews Endocrinology 12 15-28. (https://doi. org/10.1038/nrendo.2015.189)

Lawrence T \& Natoli G 2011 Transcriptional regulation of macrophage polarization: enabling diversity with identity. Nature Reviews Immunology 11 750-761. (https://doi.org/10.1038/nri3088)

Liu M, Xiang R, Wilk SA, Zhang N, Sloane LB, Azarnoush K, Zhou L, Chen H, Xiang G, Walter CA, et al. 2012 Fat-specific DsbA-L overexpression promotes adiponectin multimerization and protects mice from diet-induced obesity and insulin resistance. Diabetes $\mathbf{6 1}$ 2776-2786. (https://doi.org/10.2337/db12-0169)

Liu M, Bai J, He S, Villarreal R, Hu D, Zhang C, Yang X, Liang H, Slaga TJ, Yu Y, et al. 2014 Grb10 promotes lipolysis and thermogenesis by phosphorylation-dependent feedback inhibition of mTORC1. Cell Metabolism 19 967-980. (https://doi.org/10.1016/j. cmet.2014.03.018)

Lumeng CN \& Saltiel AR 2011 Inflammatory links between obesity and metabolic disease. Journal of Clinical Investigation 121 2111-2117. (https://doi.org/10.1172/JCI57132) 
Lumeng CN, Bodzin JL \& Saltiel AR 2007a Obesity induces a phenotypic switch in adipose tissue macrophage polarization. Journal of Clinical Investigation 117 175-184. (https://doi.org/10.1172/JCI29881)

Lumeng CN, Deyoung SM \& Saltiel AR 2007b Macrophages block insulin action in adipocytes by altering expression of signaling and glucose transport proteins. American Journal of Physiology: Endocrinology and Metabolism 292 E166-E174. (https://doi. org/10.1152/ajpendo.00284.2006)

Lumeng CN, DelProposto JB, Westcott DJ \& Saltiel AR 2008 Phenotypic switching of adipose tissue macrophages with obesity is generated by spatiotemporal differences in macrophage subtypes. Diabetes $\mathbf{5 7}$ 3239-3246. (https://doi.org/10.2337/db08-0872)

Luo Y, He F, Hu L, Hai L, Huang M, Xu Z, Zhang J, Zhou Z, Liu F \& Dai YS 2014 Transcription factor Ets1 regulates expression of thioredoxin-interacting protein and inhibits insulin secretion in pancreatic beta-cells. PLOS ONE 9 e99049. (https://doi.org/10.1371/ journal.pone.0099049)

Mantovani A, Sica A, Sozzani S, Allavena P, Vecchi A \& Locati M 2004 The chemokine system in diverse forms of macrophage activation and polarization. Trends in Immunology 25 677-686. (https://doi. org/10.1016/j.it.2004.09.015)

McGillicuddy FC, Chiquoine EH, Hinkle CC, Kim RJ, Shah R, Roche HM, Smyth EM \& Reilly MP 2009 Interferon gamma attenuates insulin signaling, lipid storage, and differentiation in human adipocytes via activation of the JAK/STAT pathway. Journal of Biological Chemistry $\mathbf{2 8 4}$ 31936-31944. (https://doi.org/10.1074/jbc.M109.061655)

McLaughlin T, Ackerman SE, Shen L \& Engleman E 2017 Role of innate and adaptive immunity in obesity-associated metabolic disease. Journal of Clinical Investigation 127 5-13. (https://doi.org/10.1172/JCI88876)

Meng W, Liang X, Chen H, Luo H, Bai J, Li G, Zhang Q, Xiao T, He S, Zhang Y, et al. 2017 Rheb inhibits beiging of white adipose tissue via PDE4D5-dependent downregulation of the cAMP-PKA signaling pathway. Diabetes 66 1198-1213. (https://doi.org/10.2337/db16-0886)

Minematsu H, Shin MJ, Celil Aydemir AB, Kim KO, Nizami SA, Chung GJ \& Lee FY 2011 Nuclear presence of nuclear factor of activated T cells (NFAT) c3 and c4 is required for Toll-like receptor-activated innate inflammatory response of monocytes/macrophages. Cell Signaling $\mathbf{2 3}$ 1785-1793. (https://doi.org/10.1016/j.cellsig.2011.06.013)

Mosser DM \& Edwards JP 2008 Exploring the full spectrum of macrophage activation. Nature Reviews Immunology 8 958-969. (https://doi.org/10.1038/nri2448)

Nevers T, Salvador AM, Velazquez F, Ngwenyama N, Carrillo-Salinas FJ, Aronovitz M, Blanton RM \& Alcaide P 2017 Th1 effector T cells selectively orchestrate cardiac fibrosis in nonischemic heart failure. Journal of Experimental Medicine 214 3311-3329. (https://doi. org/10.1084/jem.20161791)

Odegaard JI, Ricardo-Gonzalez RR, Goforth MH, Morel CR, Subramanian V, Mukundan L, Red Eagle A, Vats D, Brombacher F, Ferrante AW, et al. 2007 Macrophage-specific PPARgamma controls alternative activation and improves insulin resistance. Nature $\mathbf{4 4 7}$ 1116-1120. (https://doi.org/10.1038/nature05894)

Okabe Y \& Medzhitov R 2014 Tissue-specific signals control reversible program of localization and functional polarization of macrophages. Cell 157 832-844. (https://doi.org/10.1016/j.cell.2014.04.016)

Olefsky JM \& Glass CK 2010 Macrophages, inflammation, and insulin resistance. Annual Review of Physiology 72 219-246. (https://doi. org/10.1146/annurev-physiol-021909-135846)

Orr JS, Puglisi MJ, Ellacott KL, Lumeng CN, Wasserman DH \& Hasty AH 2012 Toll-like receptor 4 deficiency promotes the alternative activation of adipose tissue macrophages. Diabetes $612718-2727$. (https://doi.org/10.2337/db11-1595)
Osborn O \& Olefsky JM 2012 The cellular and signaling networks linking the immune system and metabolism in disease. Nature Medicine 18 363-374. (https://doi.org/10.1038/nm.2627)

Oukka M, Ho IC, de la Brousse FC, Hoey T, Grusby MJ \& Glimcher LH 1998 The transcription factor NFAT4 is involved in the generation and survival of T cells. Immunity 9 295-304. (https://doi. org/10.1016/S1074-7613(00)80612-3)

Peng SL, Gerth AJ, Ranger AM \& Glimcher LH 2001 NFATc1 and NFATc2 together control both $\mathrm{T}$ and $\mathrm{B}$ cell activation and differentiation. Immunity 14 13-20. (https://doi.org/10.1016/S10747613(01)00085-1)

Ranjan R, Deng J, Chung S, Lee YG, Park GY, Xiao L, Joo M, Christman JW \& Karpurapu M 2014 The transcription factor nuclear factor of activated T cells $\mathrm{c} 3$ modulates the function of macrophages in sepsis. Journal of Innate Immunity 6 754-764. (https://doi. org/10.1159/000362647)

Rao A, Luo C \& Hogan PG 1997 Transcription factors of the NFAT family: regulation and function. Annual Review of Immunology $\mathbf{1 5}$ 707-747. (https://doi.org/10.1146/annurev.immunol.15.1.707)

Reilly SM \& Saltiel AR 2017 Adapting to obesity with adipose tissue inflammation. Nature Reviews Endocrinology 13 633-643. (https://doi. org/10.1038/nrendo.2017.90)

Rosen ED \& Spiegelman BM 2006 Adipocytes as regulators of energy balance and glucose homeostasis. Nature 444 847-853. (https://doi. org/10.1038/nature05483)

Samuel VT \& Shulman GI 2016 The pathogenesis of insulin resistance: integrating signaling pathways and substrate flux. Journal of Clinical Investigation 126 12-22. (https://doi.org/10.1172/JCI77812)

Shaul ME, Bennett G, Strissel KJ, Greenberg AS \& Obin MS 2010 Dynamic, M2-like remodeling phenotypes of CD11c+ adipose tissue macrophages during high-fat diet - induced obesity in mice. Diabetes 59 1171-1181. (https://doi.org/10.2337/db09-1402)

Sica A, Dorman L, Viggiano V, Cippitelli M, Ghosh P, Rice N \& Young HA 1997 Interaction of NF-kappaB and NFAT with the interferon-gamma promoter. Journal of Biological Chemistry 272 30412-30420. (https://doi.org/10.1074/jbc.272.48.30412)

Stolarczyk E 2017 Adipose tissue inflammation in obesity: a metabolic or immune response? Current Opinion in Pharmacology 37 35-40. (https://doi.org/10.1016/j.coph.2017.08.006)

Vigouroux C, Caron-Debarle M, Le Dour C, Magre J \& Capeau J 2011 Molecular mechanisms of human lipodystrophies: from adipocyte lipid droplet to oxidative stress and lipotoxicity. International Journal of Biochemistry and Cell Biology 43 862-876. (https://doi. org/10.1016/j.biocel.2011.03.002)

Weisberg SP, McCann D, Desai M, Rosenbaum M, Leibel RL \& Ferrante AW Jr 2003 Obesity is associated with macrophage accumulation in adipose tissue. Journal of Clinical Investigation 112 1796-1808. (https://doi.org/10.1172/JCI200319246)

Wilkins BJ, De Windt LJ, Bueno OF, Braz JC, Glascock BJ, Kimball TF \& Molkentin JD 2002 Targeted disruption of NFATc3, but not NFATc4, reveals an intrinsic defect in calcineurin-mediated cardiac hypertrophic growth. Molecular and Cellular Biology 22 7603-7613. (https://doi.org/10.1128/MCB.22.21.7603-7613.2002)

Winer S, Chan Y, Paltser G, Truong D, Tsui H, Bahrami J, Dorfman R, Wang Y, Zielenski J, Mastronardi F, et al. 2009 Normalization of obesity-associated insulin resistance through immunotherapy. Nature Medicine 15 921-929. (https://doi.org/10.1038/nm.2001)

Zhu C, Rao K, Xiong H, Gagnidze K, Li F, Horvath C \& Plevy S 2003 Activation of the murine interleukin-12 p40 promoter by functional interactions between NFAT and ICSBP. Journal of Biological Chemistry 278 39372-39382. (https://doi.org/10.1074/jbc.M306441200)

Received in final form 21 March 2018

Accepted 1 June 2018

Accepted Preprint published online 1 June 2018
๑) 2018 Society for Endocrinology Published by Bioscientifica Ltd. Printed in Great Britain 